\title{
sciendo
}

\section{A PECULIAR CASE OF CUTANEOUS EYELID MELANOMA: CLINICOPATHOLOGICAL QUESTIONS AND THERAPEUTIC CHALLENGES}

\author{
ALIN DUMITRU CIUBOTARU ${ }^{1}$, CONSTANTIN GRIGORAȘ ${ }^{2}$, \\ CARMEN-ECATERINA LEFERMAN ${ }^{3}$
}

\author{
${ }^{1,3}$ Phd student, “Gr. T. Popa” University of Medicine and Pharmacy, Iaşi, ${ }^{2}$ Saint Spiridon County Hospital, Iași
}

\begin{abstract}
Keywords: $\quad$ malignant Abstract: Primary melanoma of the eyelid is a rare malignant tumour that accounts for $<1 \%$ of all melanoma, eyelid, malignant melanomas and about $1 \%$ of malignant tumours of the eyelid. The most common clinical cutaneous, linear presentation of melanoma is an oval lesion; linear presentation is uncommon. We presented a 79 year-old woman with a several-month history of a rapidly-growing, irregular, linear, pigmented cutaneous lesion on the right lower eyelid, involving the eyelid margin. The lesion measured $2 \mathrm{~cm} x$ $0,8 \mathrm{~cm} \times 0,2 \mathrm{~cm}$. Anterior and posterior segments appeared normal in both eyes, without pathological findings on ocular ultrasound examination. After excision biopsy, histopathological examination revealed a nodular melanoma. The case underwent additional investigations for staging and therapy. The current therapeutic strategies in eyelid melanoma include surgical excision, systemic chemotherapy, immunotherapy, targeted therapy or radiotherapy. Despite advanced research methods in the field, the management of eyelid melanoma is still a major challenge.
\end{abstract}

\section{INTRODUCTION}

Primary melanoma of the eyelid is a rare malignant tumour that accounts for $<1 \%$ of all malignant melanomas and about $1 \%$ of malignant tumours of the eyelid.(1) This tumour appears most often in the lower eyelid.(2) The most common clinical presentation of melanoma is an oval lesion, whereas linear presentation is uncommon.(2) Among histopathological subtypes of melanoma, nodular melanoma is considered the second most common form and the most aggressive variant.(3)

Due to the low incidence of the disease, there are a limited number of studies that examine the clinicopathological features and therapeutic approaches in nodular melanoma of the eyelid. Therefore, most of the actual understanding of this tumour is based on extrapolation from studies of cutaneous melanoma in other locations.

Although recent studies showed a great interest in new therapeutic methods and biopsy strategies, treatment for eyelid melanoma remains limited to surgical excision and eyelid reconstruction. $(4,5,6,7)$

\section{CASE REPORT}

A 79-year-old female patient presented to the clinic of ophthalmology with a several-month history of a rapidlygrowing, irregular, linear, pigmented cutaneous lesion on the right lower eyelid involving the eyelid margin (figure no. 1). Clinically, the lesion had a black-brown colour and irregular borders. It was ulcerated, asymmetric in two axes and covered with yellowish-brown crusts. The lesion measured $2 \mathrm{~cm} \times 0,8$ $\mathrm{cm} \times 0,2 \mathrm{~cm}$. Anterior and posterior segments appeared normal in both eyes. There were no pathological findings on ocular ultrasound examination.

An excision biopsy was performed and histopathological examination revealed a nodular melanoma with mixed morphology, epithelioid and spindle cells with a high mitotic rate $4 / \mathrm{HPF}$. It shows significant cellular pleomorphism with large nuclei and prominent eosinophilic macronuclei. The maximum tumour thickness is $3 \mathrm{~mm}$ deep (Breslow Depth $3 \mathrm{~mm}$ ), penetrating the deep dermal area and isolated muscle bundles (Clark Level IV). The limits of resection are found in healthy tissue with wide excision margins $>5 \mathrm{~mm}$. There are no aspects of vascular and perineural permeation. There was no clinical evidence of lymphadenopathy or other signs or leading symptoms of cancer. All standard laboratory tests and baseline imaging for staging were normal.

Figure no 1. Malignant melanoma of the lower eyelid at admission

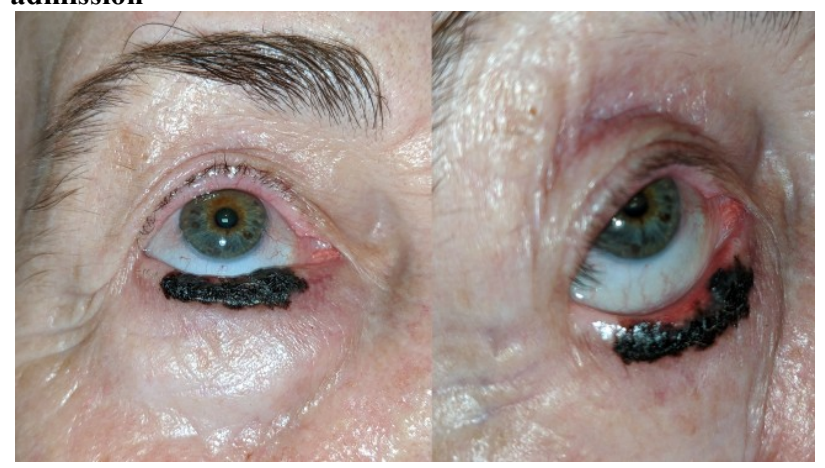

A full thickness wide excision of the lower eyelid melanoma was performed according to the International Guidelines for excision margins. The lower eyelid defect was reconstructed using free upper eyelid tarsoconjunctival and myocutaneous grafts from the patient's ipsilateral eye.

${ }^{3}$ Corresponding author: Carmen-Ecaterina Leferman, B-dul Independenței, Nr.1, Iași, România, E-mail:lefermanc@gmail.com, Phone: +40751 117413

Article received on 27.02.2020 and accepted for publication on 28.05.2020 


\section{CLINICAL ASPECTS}

There were no complications after surgery and the grafts' healing was optimal (figure no. 2.). Also, at six-month follow-up, the evaluation revealed no abnormality.

Figure no. 2. Postoperative outcome after a. 1 day b. 4 days c. 2 weeks d. 6 months

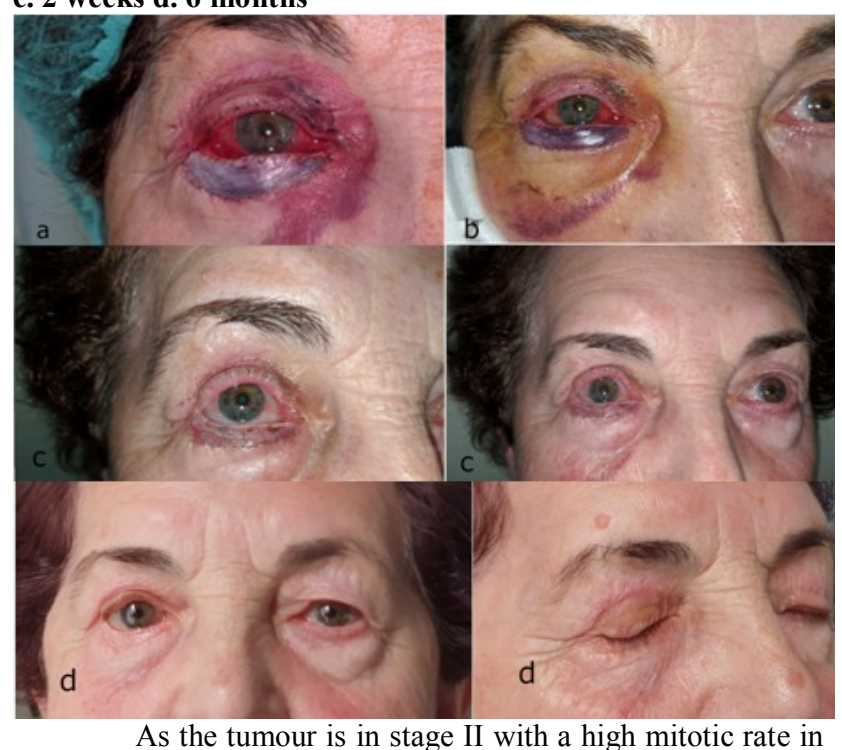

an older age patient, the prognosis tends to be less favourable.

DISCUSSIONS

Cutaneous eyelid melanoma is a malignancy of melanocytes, the pigment producing cells within the skin. There are 4 histopathological types of cutaneous melanoma: superficial spreading melanoma (SSM), nodular melanoma (NM), lentigo maligna melanoma and acral lentiginous melanoma. The most common histopathological type is SSM and the second common is NM (3), the form presented by the patient in this report. Melanoma cells can be classified in 2 types: epithelioid and spindle cells. Typically, NM presents large epithelioid cells with abundant eosinophilic cytoplasm, large nuclei and prominent macronuclei, spindle cell melanoma being a rare subtype of malignant melanoma.(1) The tumour from the patient in this report has both histopathological features. It is a mixed epithelioid and spindle cell melanoma. The mixed morphologic variant is less frequent and characteristic for male higher-age patients.(8)

Another particularity of the case is the clinical aspect as a linear lesion. Mostly, melanoma is round or oval shaped.(2) Only two cases of linear melanoma in situ on the trunk were described in the literature.(9) However, to the best of my knowledge, the female in this report is the first patient with a linear nodular melanoma of the eyelid to be described in the literature. Although, there is a linear progression model of melanoma (10), it is possible that additional cases with primary cutaneous melanomas linear tumours have been observed, but the details of these patients have not been published.

Linear model of melanoma progression is a controversial concept. Via linear model, melanoma starts with the transformation of epidermal melanocytes that initially has radial growth specific for SSM and subsequently turns into vertical growth, characteristic to NM.(10) According to this concept, SSM and NM arise sequentially. Now, the question arises: if the tumour of the patient in the report was firstly SSM and an early detection could radically change the prognostic and therapeutic strategy. However, there are clinical and biological data that suggest that these 2 types are distinct entities.(10) On the other hand, this linear pattern could be explained by the influence of Blaschko and Langer tension lines on the skin.

The classification of melanoma subtypes has prognostic and therapeutic importance. Recent studies show a great interest in new therapeutic methods and biopsy strategies. The type of treatment depends on the stage and the location of the tumour.

The current therapeutic strategies in eyelid melanoma include surgical excision, chemotherapy, immunotherapy, targeted therapy or radiotherapy. $(5,6,7,11)$ In stage I eyelid melanoma, wide local excision is the first choice and most often, no other treatment is needed.(12) Moreover, studies show that Mohs surgery (12) or Imiquimod cream could be used.(13) In stage II and especially in stage III, beside wide excision, sentinel lymph node biopsy (SLNB) is recommended, but there is not enough data about the relationship between SLN status and patient survival. Through the years, more clinical and histological criteria have been included for patient selection: tumours $\geq 1 \mathrm{~mm}$ thick, Clark level $\geq \mathrm{IV}$, tumours with high mitotic rate $>1$ mitotic/HPF, or those with histologic ulceration. However, SLNB is still not universally applied in the management of the periocular melanoma.(14) If SLNB is positive, adjuvant therapy is needed. Immune checkpoint inhibitors or targeted therapy drugs (for tumours with BRAF gene mutations) are used in melanoma treatment.(11) Another option is radiation therapy.(15) If SLNB is negative, close follow up is important. In stage IV melanoma symptomatic treatment is often needed. Also, patients can be included in clinical trials.(16)

Due to the anatomic particularities of the eyelid, the reconstruction of the defect after surgical excision of the neoplasm is another challenge. For long term aesthetic and functional results there are different techniques. In this case, we used a procedure that has the advantage of avoiding multi staged techniques, with good cosmetic and functional results, according to International Guidelines for excision margins.

CONCLUSIONS
The linear appearance of cutaneous melanoma is an extremely rare form of presentation that can lead to misdiagnosis and late presentation to the hospital, with prognostic and therapeutic consequences. In the management of an eyelid melanoma, two aspects must be highly considered in order to achieve an optimal balance between cosmetic and curative results: tumour aggressiveness and the particularities of anatomic and functional structures that are involved. The linear lesions in the early stage can be included in differential diagnoses of melanoma in situ.

\section{REFERENCES}

1. Boulos PR, Rubin PAD. Cutaneous melanomas of the eyelid. Semin Ophthalmol. 2006 Sep;21(3):195-206.

2. Higgins HW, Lee KC, Galan A, Leffell DJ. Melanoma in situ: Part I. Epidemiology, screening, and clinical features. J Am Acad Dermatol. 2015 Aug;73(2):181-90, quiz 191-2.

3. Porras BH, Cockerell CJ. Cutaneous malignant melanoma: classification and clinical diagnosis. Semin Cutan Med Surg. 1997 Jun;16(2):88-96.

4. Beaulieu D, Fathi R, Srivastava D, Nijhawan RI. Current perspectives on Mohs micrographic surgery for melanoma. Clin Cosmet Investig Dermatol. 2018;11:309-20.

5. Silverman N, Shinder R. What's New in Eyelid Tumors Asia-Pac J Ophthalmol Phila Pa. 2017 Apr;6(2):143-52.

6. Mattia G, Puglisi R, Ascione B, Malorni W, Carè A, Matarrese P. Cell death-based treatments of melanoma:conventional treatments and new therapeutic strategies. Cell Death Dis. 2018 25;9(2):112.

7. Memarzadeh K, Engelsberg K, Sheikh R, Malmsjö M. 
Large Eyelid Defect Repair Using a Free Full-Thickness Eyelid Graft. Plast Reconstr Surg Glob Open. 2017 Jul 26;5(7).

8. Xu Z, Shi P, Yibulayin F, Feng L, Zhang H, Wushou A. Spindle cell melanoma: Incidence and survival, 1973-2017. Oncol Lett. 2018 Oct;16(4):5091-9.

9. Cohen PR. Linear Malignant Melanoma In Situ: Reports and Review of Cutaneous Malignancies Presenting as Linear Skin Cancer. Cureus. 2017 Sep;9(9).

10. Greenwald HS, Friedman EB, Osman I. Superficial spreading and nodular melanoma are distinct biological entities: a challenge to the linear progression model. Melanoma Res. 2012 Feb;22(1):1-8.

11. Dummer R, Brase JC, Garrett J, Campbell CD, Gasal E, Squires $\mathrm{M}$, et al. Adjuvant dabrafenib plus trametinib versus placebo in patients with resected, BRAFV600 mutant, stage III melanoma (COMBI-AD): exploratory biomarker analyses from a randomised, phase 3 trial. Lancet Oncol. 2020 Jan 30.

12. Rosko AJ, Vankoevering KK, McLean SA, Johnson TM, Moyer JS. Contemporary Management of Early-Stage Melanoma: A Systematic Review. JAMA Facial Plast Surg. 2017 May 1;19(3):232-8.

13. Verga E, Chohan B, Verdolini R. Malignant Melanoma Treated with Topical Imiquimod: A Bespoke Treatment That Spared the Amputation. Case Rep Dermatol. 2019 Apr;11(1):1-6.

14. Mendoza PR, Grossniklaus HE. Sentinel lymph node biopsy for eyelid and conjunctival tumors: what is the evidence? Int Ophthalmol Clin. 2015;55(1):123-36.

15. Lazarevic $\mathrm{D}$, Ramelyte $\mathrm{E}$, Dummer $\mathrm{R}$, Imhof $\mathrm{L}$. Radiotherapy in Periocular Cutaneous Malignancies: A Retrospective Study. Dermatol Basel Switz. 2019;235(3):234-9.

16. Knackstedt T, Knackstedt RW, Couto R, Gastman B. Malignant Melanoma: Diagnostic and Management Update. Plast Reconstr Surg. 2018;142(2):202e-16e. 